\title{
Valor pronóstico de la monitoría ambulatoria de presión arterial en pacientes hipertensos: Observaciones en la literatura
}

\author{
Paul Mc-Nab M, Jorge E Jalil.
}

\author{
Prognostic value of Ambulatory \\ Blood Pressure Monitoring in \\ hypertensive patients. A review
}

There is a large amount of information in the literature to state that blood pressure control using ambulatory blood pressure measurement has a better predictive value for future cardiovascular events and target organ damage than casual blood pressure measurements. Clinical studies are currently evaluating if this approach is costeffective. At the present time, and in the usual clinical practice, the use of ambulatory blood pressure monitoring is recommended for patients with resistant or difficult-to-control hypertension, for hypertensive patients with previous cardiovascular events or significant target organ damage, for the diagnosis of white coat hypertension, when there are symptoms suggesting hypotension or there is large variability in casual blood pressure measurements (Rev Méd Chile 2005; 133: 89-95).

(Key-words: Blood pressure, high; Blood pressure monitoring, ambulating; Hypertension)

Recibido el 29 de junio, 2004. Aceptado en versión corregida el 16 de noviembre, 2004.

Departamento de Enfermedades Cardiovasculares. Pontificia Universidad Católica de Chile.

$\mathrm{D}$ esde hace más de 100 años, el método para el control habitual de la presión arterial (PA) ha sido el esfigmomanómetro de mercurio ${ }^{1}$. Progresivamente, se ha ido reconociendo que los valores de $\mathrm{PA}$, así medidos, no representarían fielmente la PA real, por distintas razones: tendencia al redondeo de valores, variabilidad de la PA durante el día o por el efecto de «delantal blanco $\imath^{2,3}$. De hecho, el coeficiente de correlación

Correspondencia a: Dr. Jorge Jalil. Pontificia Universidad Católica de Chile. Departamento de Enfermedades Cardiovasculares. Lira 85 Segundo Piso, Santiago. Fax 6338574. E-mail: jjalil@med.puc.cl de la PA medida en la consulta, pocas veces es mayor de 0,5 respecto a las presiones arteriales promedio, obtenidas mediante monitorización ambulatoria de la presión arterial (MAPA) ${ }^{4-6}$.

Por esto ha habido un interés progresivo, tanto clínico como en investigación clínica, en el uso de la MAPA, que al efectuar múltiples mediciones de PA durante las $24 \mathrm{~h}$, puede reflejar más exactamente la PA real de un sujeto. Adicionalmente, es posible caracterizar mejor la eficacia del tratamiento antihipertensivo durante las $24 \mathrm{~h}$, lo que podría facilitar un manejo clínico más efectivo ${ }^{7}$.

Durante los últimos años, la mayor experiencia con el uso de la MAPA, ha generado recomen- 
daciones de las distintas sociedades médicas acerca de su uso, con lo que se ha podido estandarizar su empleo ${ }^{7-11}$.

Con el objeto de evaluar la importancia de la MAPA en el control habitual de los pacientes hipertensos, hemos revisado la literatura existente sobre su utilidad y resultados en pacientes hipertensos, concentrándonos en la información que proporciona la MAPA en relación a la repercusión en órganos blanco y al riesgo de eventos cardiovasculares.

\section{PROMEDIO DE PRESIÓN ARTERIAL EN $24 \mathrm{H}$}

Uno de los estudios más relevantes en este aspecto es el estudio SAMPLE, en el que se evaluaron 206 pacientes hipertensos con una edad promedio de $50 \pm 9$ años e hipertrofia ventricular izquierda (HVI), definida como un índice de masa ventricular izquierda por ecocardiografía mayor de $110 \mathrm{~g} / \mathrm{m}^{2}$ en mujeres y de $136 \mathrm{~g} / \mathrm{m}^{2}$ en hombres, los cuales fueron tratados con inhibidores de la enzima convertidora de angiotensina (ECA) y diuréticos, con seguimiento de 12 meses. Se demostró que los promedios de PA sistólica y diastólica de la MAPA se correlacionaron mejor con la regresión de la HVI que los controles habituales de PA ( $r=0,42$ y $r=0,38$ respectivamente, $\mathrm{p}<0,01)^{12}$.

La superioridad de la MAPA en la predicción de accidente vascular encefálico (AVE) y de eventos cardiovasculares (CV) (insuficiencia cardíaca, infarto al miocardio o muerte súbita) fue demostrada a través de un subestudio del Syst-Eur, donde se incluyeron 808 pacientes mayores de 60 años con hipertensión sistólica aislada en la MAPA (PA sistólica entre 160 y $219 \mathrm{mmHg}$ con PA diastólica menor de $95 \mathrm{mmHg}$ ). Del total, se trataron aleatoriamente 415 pacientes con nitrendipino (10-40 $\mathrm{mg} / \mathrm{d}$ ), con la posibilidad de adicionar enalaprila (5-20 mg/d) o hidroclorotiazida $(12,5-25 \mathrm{mg} / \mathrm{d})$; a los 393 pacientes restantes les fue administrado placebo. Durante el seguimiento de 4,4 años, 98 pacientes presentaron eventos CV. Después del ajuste por edad, género, PA en la consulta, tratamiento recibido, eventos previos y tabaquismo, la PA sistólica promedio nocturna fue predictora de eventos CV y de AVE. El aumento del promedio de la PA sistólica nocturna en $10 \mathrm{mmHg}$ en la MAPA, aumentó el riesgo combinado de AVE o de eventos $\mathrm{CV}$, definidos como falla cardíaca fatal o no fatal, infarto al miocardio fatal o no fatal y muerte súbita, a $1,2(1,08-1,35 ; p \leq 0,01)$ siendo el incremento del riesgo de eventos CV y de AVE 1,16 (1,02-1,33; $p$ $\leq 0,05)$ y $1,31(1,02-1,33 ; \mathrm{p} \leq 0,05)$ respectivamente. No se obtuvieron diferencias significativas al estudiar las presiones sistólicas diurnas promedio. La PA sistólica promedio de 24 horas se asoció con un riesgo de $A V E$ de 1,36 (1,04-1,79; $p \leq 0,01)$. El riesgo combinado de AVE y eventos CV fue de 1,17 (1,011,35; $\mathrm{p} \leq 0,05)^{13}$.

Otro estudio relevante fue dirigido por Redon, quien estudió 86 pacientes con una edad promedio de $53 \pm 9$ años (27 hombres y 59 mujeres, hipertensos refractarios, con presiones diastólicas $>100 \mathrm{mmHg}$ en los controles, a pesar de un tratamiento antihipertensivo con 3 o más fármacos, que incluían un diurético). Los pacientes fueron seguidos por un promedio de 4 años y se evaluó: a) la aparición de repercusión sobre órganos blanco y b) eventos cardiovasculares (infarto al miocardio, angina, revascularización coronaria, AVE, isquemia cerebral transitoria, muerte súbita, enfermedad arterial oclusiva aortoilíaca, falla cardíaca progresiva 0 emergencia hipertensiva). Durante el seguimiento, 21 pacientes presentaron un primer evento cardiovascular. Después de ajustar por edad, género, tabaquismo, hipertrofia ventricular izquierda y PA medida en consulta, el número de eventos fue significativamente más alto en el tercil superior de la presión diastólica promedio diurna (13,6 eventos por 100 pacientes-año $)(p<0,02)$ en relación al tercil central (9,5 eventos por 100 pacientes-año) y el tercil inferior (2,2 eventos por 100 pacientes-año). Respecto al daño sobre órganos blanco, se encontró una diferencia estadísticamente significativa en el tercil superior de la presión diastólica promedio diurna ( $p<0,03$ ), no así en los otros grupos. A pesar del bajo número de pacientes y la falta de ajuste estadístico para los niveles de colesterol e historia familiar de cardiopatía coronaria, este fue el primer estudio que demostró el valor de la MAPA en el control de pacientes hipertensos resistentes. De acuerdo a este estudio, los hipertensos con promedio de PA diastólica durante el día mayor o igual a $88 \mathrm{mmHg}$ y con controles en 
la consulta con presiones mayores de $100 \mathrm{mmHg}$, a pesar de tratamiento con 3 o más fármacos antihipertensivos, tendrían un peor pronóstico futuro ${ }^{14}$.

Otro estudio relevante fue realizado por Verdecchia y cols, quienes evaluaron 790 pacientes hipertensos sin tratamiento antihipertensivo previo, con un promedio de edad de 51 años (56\% hombres). En el seguimiento, por un promedio de 3,7 años, la MAPA predecía mejor el riesgo de eventos CV (infarto al miocardio, angina inestable, insuficiencia cardíaca que requiriera hospitalización, AVE e isquemia cerebral transitoria) que el control de PA habitual en la consulta ${ }^{15}$. Observaron que el aumento de $12 \mathrm{mmHg}$ en la PA sistólica promedio de $24 \mathrm{~h}$ aumentaba el riesgo de futuros eventos CV en $49 \%$ ( $p=0,003)$, y el aumento de 8 $\mathrm{mmHg}$ en la PA diastólica promedio de $24 \mathrm{~h}$ aumentaba el riesgo de eventos CV en $47 \%$ ( $p$ $\leq 0,01$; Figura 1). La PA obtenida en la consulta no se correlacionó con fututos eventos CV $(\mathrm{RR} 0,63)$ (0,31-1,31).

Más recientemente, Clement et al, estudiaron prospectivamente, 1.963 pacientes hipertensos tratados (edad promedio $56 \pm 13$ años; 52\% hombres), por un promedio de 5 años, donde, comparando el valor de realizar 3 tomas de PA en la consulta versus la MAPA, se observó que la MAPA predecía en forma más exacta el riesgo futuro de eventos CV (AVE, IMA, ICC, muerte súbita, angina estable y enfermedad arterial oclusiva). Al aumentar en una desviación estándar la PA sistólica y diastólica promedio de $24 \mathrm{~h}$, aumentaba el riesgo de eventos CV en 1,34 (intervalo de confianza 95\%, 1,1-1,62) y 1,21 (IC 95\%, 1,01-1,46) respectivamente, previo ajuste por la PA de la consulta al momento del ingreso ${ }^{16}$.

Kario et al, estudiaron 147 sujetos normotensos con una edad promedio de 68 años (38\% hombres) y 811 pacientes hipertensos con una edad promedio de 73 años (37\% hombres), quienes se controlaron con MAPA previa suspensión de la terapia antihipertensiva por al menos 14 días $\mathrm{y}$, posteriormente, fueron seguidos durante un promedio de 3,4 años. El riesgo de AVE fue 2\% en el grupo normotenso y $2,1 \%$ en el grupo de hipertensos que tenían promedios de PA de $24 \mathrm{~h}$ menores de 130/80 mmHg en la MAPA. El grupo de hipertensos con promedios de PA en $24 \mathrm{~h}$ mayores que $130 / 80$ en la MAPA al ingreso presentaba un riesgo de AVE de $9,4 \%$ en el seguimiento $(p<0,01)$. En este último grupo de pacientes, $61 \%$ se encontraba bajo tratamiento antihipertensivo a discreción del médico tratante (diuréticos, alfa o betabloqueadores, calcio antagonistas o inhibidores de la ECA). Durante el seguimiento no hubo controles periódicos con MAPA, no se estimó el poder pronóstico de la PA en la consulta ni fue analizada la respuesta a la terapia antihipertensiva ${ }^{17}$.

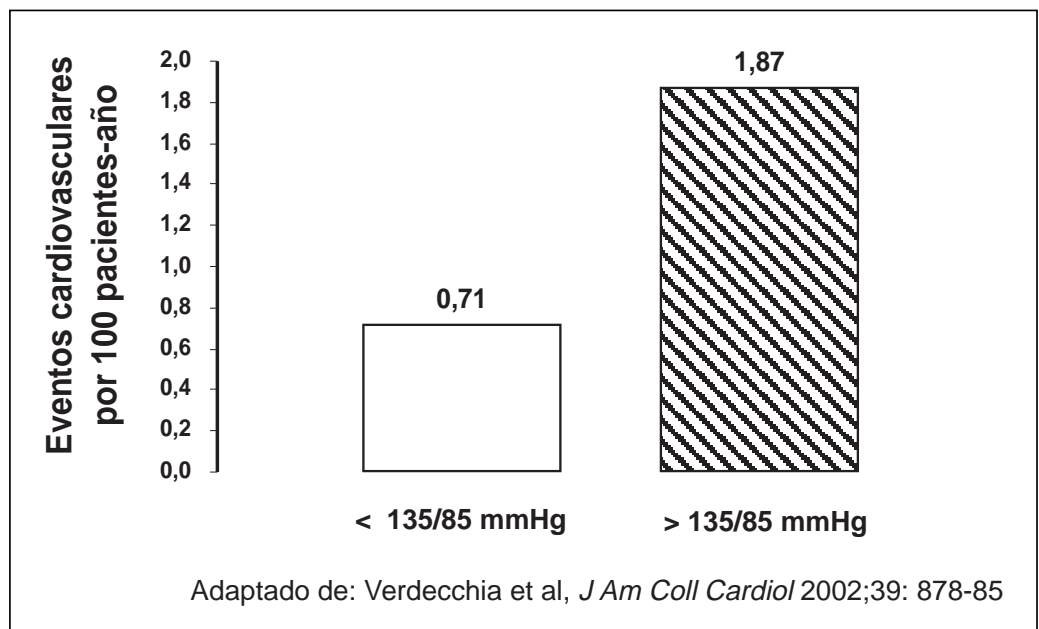

Figura 1. Incidencia de eventos cardiovasculares en pacientes hipertensos según presión arterial promedio diurna. 


\section{PRESIÓN ARTERIAL NOCTURNA}

Normalmente la PA desciende durante la noche, fenómeno que está preservado en la mayoría de los pacientes hipertensos ${ }^{18,19}$. El descenso nocturno de la PA promedio ha llevado a varios autores a categorizar los pacientes en 2 grupos: los que presentan una caída menor al 10\% respecto a la PA promedio diurna (non-dippers), en los habría mayor daño en los órganos blanco que en el grupo que presenta una caída mayor al 10\% durante la noche (〈dippers»), clasificación que fue introducida por primera vez por O'Brien et $\mathrm{al}^{20}$.

Este fenómeno ha sido debatido en la literatura por su falta de reproducibilidad en el tiempo y por el hecho de dicotomizar en forma arbitraria una variable que es continua. En un subestudio del estudio SAMPLE ya mencionado, $40 \%$ de los pacientes (sin tratamiento o con tratamiento estable) se intercambiaron entre estas 2 categorías («dipper»a «non-dipper»y viceversa) al repetirse el análisis y sin mediar un factor conocido, al comparar la evaluación a los 3 y a los 12 meses de seguimiento. En este estudio el hecho de ser «dipper» o «on-dipper» no se correlacionó con el grado de HVI al año de control ${ }^{21}$.

Ohkubo et al, comunicaron datos contradictorios con el estudio SAMPLE. Siguieron un grupo de 1.542 personas residentes en Ohasama, Japón, por un promedio de 9,2 años, de las cuales, $21 \%$ eran sujetos hipertensos (con tratamiento farmacológico sólo $13 \%$ de este grupo). Se observó que el grupo «non-dipper» (que incluyó sujetos hipertensos y no hipertensos) presentaba un riesgo relativo de muerte de causa CV de 2,16 (1,31-3,55; p $<0,01)$. En el grupo de sujetos hipertensos el riesgo fue 2,37 (1,49-3,75; $p<0,02)$. En el grupo de pacientes hipertensos y unon-dippers» el riesgo de muerte de causa CV era de $5,37(2,62-11 ; p=0,0001)$. Este estudio tiene la limitación de la falta de ajuste por diabetes 0 dislipidemia $^{22}$ (Figura 2).

Yamamoto et al estudiaron 105 pacientes en Kioto, Japón, con una edad promedio de 67 años, de los cuales 68\% eran hombres, con AVEs lacunares evaluados con MAPA y resonancia nuclear magnética, seguidos por un promedio de 3,2 años. Observaron que el grupo que presentaba un menor grado de disminución de la PA durante la noche, presentaba mayor riesgo de recurrencia de AVE (1,3\% para la PA sistólica y 3,3\% para PA diastólica). En cambio, el grupo que no presentaba nuevos AVE, tenía mayores modificaciones en la presión arterial entre el día y la noche $(7,2 \%$ para la presión sistólica y 10,4\% para la presión diastólica) ${ }^{23}$.

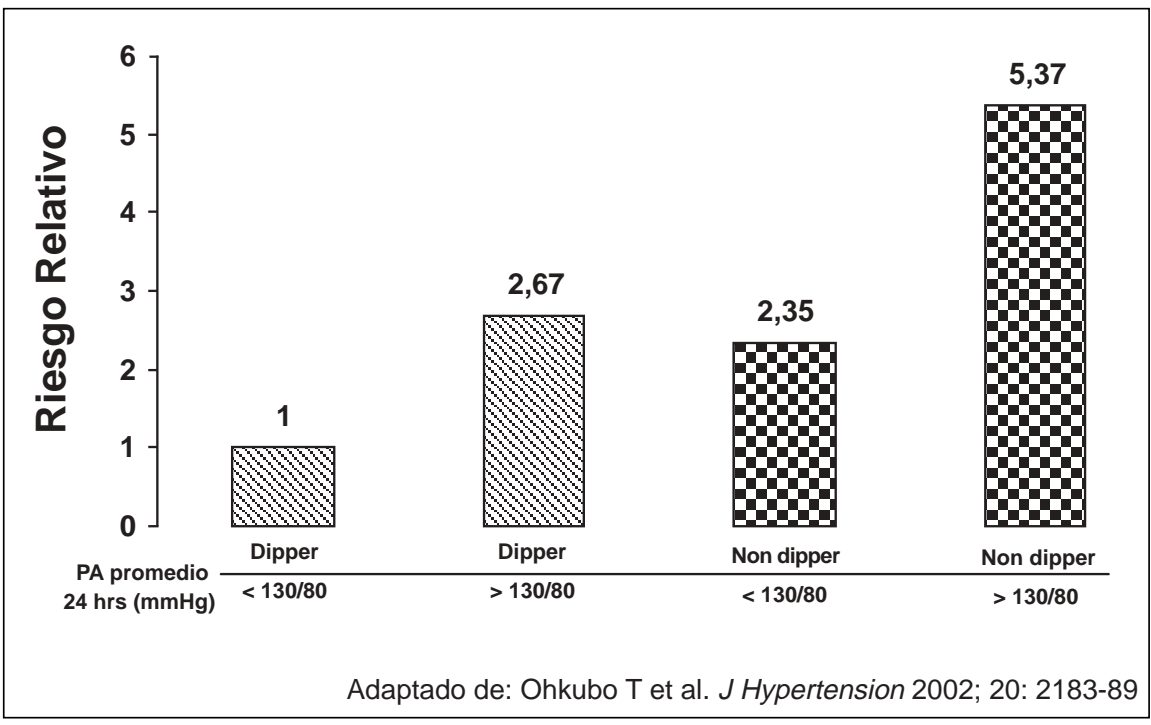

FIGURA 2. Riesgo relativo de muerte de causa cardiovascular según descenso de la PA nocturna (dipper vs nondipper) y PA promedio de $24 \mathrm{~h}$. 
En el análisis del estudio Syst-Eur mencionado previamente, la relación noche/día de la PA sistólica promedio fue un predictor independiente de riesgo de eventos $\mathrm{CV}$ en el subgrupo propuesto a recibir placebo. Por cada $10 \%$ de aumento de la relación noche/día de la presión sistólica promedio, el riesgo de nuevos eventos CV aumentaba a 1,41 (1,03-1,94; $\mathrm{p} \leq 0,05)$, después del control por múltiples factores confundentes (incluida la PA promedio de 24 horas). Además, en el grupo placebo observaron que la PA sistólica promedio nocturna era un mejor predictor de todos los end-points en 1,35 que la PA sistólica promedio diurna $(1,1-1,66 ; \quad p$ $\leq 0,01)^{13}$.

Debido a lo anterior, parece haber evidencias importantes que indican que la medición de la relación de la PA del día versus la noche es un elemento importante en el control de los hipertensos, ya que ayuda a estratificar mejor el riesgo cardiovascular futuro, por sobre del control habitual de la presión y de los otros marcadores pronósticos tradicionales.

\section{PRESIÓN DE PULSO AMBULATORIA}

Diversos estudios han mostrado una correlación entre los niveles de presión de pulso (PP), o diferencia entre las PA sistólica y diastólica, y el riesgo cardiovascular futuro, independiente de la PA sistólica y diastólica ${ }^{24-26}$. Sin embargo, la PP se ve afectada por la reacción de alerta, por lo que los valores medidos en la consulta médica habitual no serían concluyentes. Mancia et $\mathrm{al}^{26}$, evaluaron a 48 pacientes, con edades entre 17 y 67 años ( $54 \%$ hombres) y demostraron, midiendo la PA intraarterial, un aumento promedio de 12 $\mathrm{mmHg}$ de la PP (rango de 4-75 $\mathrm{mmHg}$ en PA sistólica y de 1-36 mmHg en PA diastólica) respecto a los momentos previos a la evaluación médica.
Para evaluar la importancia de la PP, Verdecchia et al evaluaron 2.010 pacientes hipertensos del estudio PIUMA, con una edad promedio de 51,7 años (52\% hombres), sin tratamiento y sin complicaciones, seguidos por un promedio de 3,8 años. El riesgo de eventos CV por 100 pacientesaño en los tres terciles de distribución del promedio de presión de pulso durante las $24 \mathrm{~h}$, fue 1,19; 1,81 y 4,92 y para los eventos fatales fueron 1,11 ; 1,17 y 1,23 ( $p<0,01$ ). El riesgo de eventos CV al evaluar la PP de la PA tomada en la consulta fue 1,$38 ; 2,12$ y 4,34 para el primer, segundo y tercer tercil, respectivamente ${ }^{24}$.

Por lo anterior, habría argumentos para afirmar que la PP es un elemento adicional para precisar el riesgo CV de los pacientes hipertensos. De acuerdo a un planteamiento reciente, los sujetos hipertensos con mayor riesgo cardiovascular, serían aquellos que, además de HTA diagnosticada con la MAPA, son «non-dippers» y/o tienen una PP promedio en 24 horas $>53$ $\mathrm{mmHg}^{27}$.

\section{CONCLUSIONES}

Existe numerosa información, en la literatura médica, para afirmar que el control de la PA con MAPA predice mejor los eventos CV futuros y la repercusión sobre los órganos blanco, que las determinaciones casuales de la PA. Hay estudios en curso para definir si esto puede ser beneficioso en relación al costo. Por el momento, y en el contexto de la práctica clínica habitual, su uso está recomendado para pacientes hipertensos de difícil control o resistentes, o que ya han presentado eventos CV o presentan repercusión importante sobre órganos blanco; en el diagnóstico de la hipertensión de delantal blanco; cuando existen síntomas sugerentes de hipotensión arterial y cuando hay una variabilidad importante en las cifras de presión arterial. 


\section{REFERENCIAS}

1. Chavez Dominguez R, de Michel A. Epistemological focus on sphygmomanometry. Rev Invest Clin 2002; 54: 84-91.

2. Verdecchia $\mathrm{P}$, SchiLACI G, Borgioni C, Ciucci A, Zampi I, Gattobigio R et al. White coat hypertension and white coat effect. Similarities and differences. Am J Hypertension 1995; 8: 790-8.

3. Hessel PA. Terminal digit preference in blood pressure measurements: effects on epidemiological associations. Int J Epidemiol 1986; 15: 122-5.

4. Mancia G, Omboni S, Ravogli A, Parati G, Zanchetti A. Ambulatory blood pressure monitoring in the evaluation of antihypertensive treatment: additional information from a large data base. Blood Press 1995; 4: 148-56.

5. Mancia G, Sega R, Bravi C, De Vito G, Valagussa F, Cesana G, ZanchetTi A. Ambulatory blood pressure normality: results from the PAMELA study. J Hypertension 1995; 13: 1377-90.

6. Imai $Y$, Nagai $K$, Sakuma M, Sakuma $H$, Nakatsuka $H$, SАтон H Eт AL. Ambulatory blood pressure of adults in Ohasama, Japan. Hypertension 1993; 22: 900-12.

7. O’Brien E, Coats A, Owens P, Petrie J, Padfield PL, LTTLER WA ET AL. Use and interpretation of ambulatory blood pressure monitoring: recommendations of the British Hypertension Society. BMJ 2000; 320: 1128-34.

8. The sixth report of the Joint National Committee on prevention, detection, evaluation, and treatment of high blood pressure. Arch Intern Med 1997; 157: 2413-46.

9. ZANChetTI A. The role of ambulatory blood pressure monitoring in clinical practice. Am J Hypertension 1997; 10: 1069-80.

10. Prat H, Valdés G, Román O, Zárate lH, Jalll J. [Recommendations for the use of ambulatory blood pressure monitoring. Consensus document of the Chilean Hypertension Society]. Rev Méd Chile 1999; 127: 1269-73.

11. Staessen JA, Beilin L, Parati G, Waeber B, White W. Task force IV: Clinical use of ambulatory blood pressure monitoring. Participants of the 1999 Consensus Conference on Ambulatory Blood Pressure Monitoring. Blood Press Monit 1999; 4: 319-31.
12. Mancia G, Zanchetti A, Agabiti-Rosei E, Benemio G, De Cesaris R, Fogari R et al. Ambulatory blood pressure is superior to clinic blood pressure in predicting treatment-induced regression of left ventricular hypertrophy. SAMPLE Study Group. Study on Ambulatory Monitoring of Blood Pressure and Lisinopril Evaluation. Circulation 1997; 95: 1464-70.

13. Staessen JA, Thijs L, Fagard R, O’Brien ET, Clement D, DE LeEUW PW ET AL. Predicting cardiovascular risk using conventional vs ambulatory blood pressure in older patients with systolic hypertension. Systolic Hypertension in Europe Trial Investigators. JAMA 1999; 282: 539-46.

14. Redon J, Campos C, Narciso ML, Rodicio JL, Pascual JM, Ruilope LM. Prognostic value of ambulatory blood pressure monitoring in refractory hypertension: a prospective study Hypertension 1998; 31: 712-8.

15. Verdecchia P, Reboldi G, Porcellati $C$, Schilaci G, Pede S, Bentivoglio M et al. Risk of cardiovascular disease in relation to achieved office and ambulatory blood pressure control in treated hypertensive subjects. J Am Coll Cardiol 2002; 39: 878-85.

16. Clement DL, De Buyzere ML, De Bacquer DA, de Leeuw PW, Duprez DA, Fagard RH et al. Office versus Ambulatory Pressure Study Investigators. Prognostic value of ambulatory blood-pressure recordings in patients with treated hypertension. N Engl J Med 2003; 348: 2407-15.

17. Kario K, Shimada K, Schwartz JE, Matsuo T, Hoshide S, PICKERING TG. Silent and clinically overt stroke in older Japanese subjects with white-coat and sustained hypertension. J Am Coll Cardiol 2001; 38: 238-45.

18. Staessen JA, Bieniaszewski L, O’Brien E, Gosse P, Hayashi H, Imai Y et al. Nocturnal blood pressure fall on ambulatory monitoring in a large international database. The «Ad Hoc» Working Group. Hypertension 1997; 29: 30-9.

19. PICKeRING TG. The clinical significance of diurnal blood pressure variations. Dippers and nondippers. Circulation 1990; 81: 700-2.

20. O’Brien E, Sheridan J, O'Maley K. Dippers and non-dippers. Lancet 1988; 2: 397.

21. Omboni S, Parati G, Palatini P, Vanasia A, Muiesan ML, Cuspidi C, Mancia G. Reproducibility and clinical value of nocturnal hypotension: prospec- 
tive evidence from the SAMPLE study. Study on Ambulatory Monitoring of Pressure and Lisinopril Evaluation. J Hypertension 1998; 16: 733-8.

22. Оhкubo T, Hozawa A, Yamaguchi J, Kikuya M, Ohmori K, Michimata M et al. Prognostic significance of the nocturnal decline in blood pressure in individuals with and without high 24-h blood pressure: the Ohasama study. J Hypertension 2002; 20: 2183-89.

23. Yamamoto Y, AKiguchi I, Oiwa K, Hayashi M, Kimura $\mathrm{J}$. Adverse effect of nighttime blood pressure on the outcome of lacunar infarct patients. Stroke 1998; 29: 570-6.

24. Verdecchia P, Schilaci G, Borgioni C, Ciucci A, Pede S, Porcelati C. Ambulatory pulse pressure: a potent predictor of total cardiovascular risk in hypertension. Hypertension 1998; 32: 983-8.

25. Benetos A, Safar M, Rudnichi A, Smulyan H, Richard JL, Ducimetieere P, Guize L. Pulse pressure: a predictor of long-term cardiovascular mortality in a French male population. Hypertension 1997; 30: 1410-15.

26. Blacher J, Staessen Ja, Girerd X, Gasowski J, Thijs L, LU L ET AL. Pulse pressure not mean pressure determines cardiovascular risk in older hypertensive patients. Arch Intern Med 2000; 160: 1085-9.

27. Verdecchia P, Angel F, Gattobigio R. Clinical Usefulness of Ambulatory Blood Pressure Monitoring. J Am Soc Nephrol 2004; 15: S30-S33. 